\title{
Structural Optimization and Measurement of Chinese Employees' Perception of Being Trusted
}

\author{
DING Yue lan ${ }^{1}$, LIANG Zhizhuo ${ }^{2 *}$ \\ International Business School of Shaanxi Normal University \\ Xi'an, Shaanxi, China
}

\begin{abstract}
In order to develop an employee perception of being trusted scale based on our cultural background ,in-depth interviews with seven state-owned enterprises and six privatelyowned enterprise personnel; using the grounded theory to construct a structural model of employees' perception of being trusted; exploratory factor analysis and confirmatory factor analysis were used to develop the scale; which ultimately established the three dimensions of competence recognition, establishment of intimate relationships, and leadership support. The theoretical structural model was verified and developed the scale that have typical Chinese cultural characteristics, which provides a research tool for continuing to explore the perception of being trusted of employees in China.
\end{abstract}

Keywords-Perception of being trusted; Grounded theory; Structural model; Scale development

\section{INTRODUCTION}

Perception of being trusted is a new type of trust. Researchers define it as one side's perception of the willingness to undertake the risks of another side. For managers, "a gentleman will die for the patron who recognizes his worth, a girl will doll herself up for the man who loves her", only when employees perceive that they are being trusted, will the influence of the superiors' trust on their behaviors be shown [2]. For employees, the feeling of being trusted is a psychological authorization, and can improve work achievement through influencing responsibility standardization [3], the sense of self-respect in organization and organization commitment [4]; and may also cause role load and work pressure, leading to emotional exhaustion [5] or form compulsory citizen's behaviors [6]. Therefore, the perception of being trusted has unique and important effects on employees' behaviors and achievement, accurately grasping the structure of Chinese employees' perception of being trusted and measuring employees' degree of their perception of being trusted have very important significance for predicting employees' behaviors.

Through researches on the scales of the perception of being trusted at home and abroad, it is found that problems in the selection of the scales of the perception of being trusted according to the current condition in China are as follows: (1) Most of the existing scales coming from abroad are varied, and there is no unified standard of use. (2) No independent factor that can show China's special culture is found in the existing scales. Scales developed on the basis of Western culture are not necessarily applicable for Chinese employees and can not necessarily accurately measure the degree to which Chinese employees perceive that they are trusted. (2) The existing scales mainly revise and rectify scales of trusting others, there is no direct, deep exploration of the issue of how the employees perceive that they are trusted, which may influence the truthfulness of the measurement.

Therefore, this research uses the qualitative research method constructs the model of the structure of employees' perception of being trusted; Through the questionnaire and empirical analysis of the theoretical model to verify the cognition of our employees perceived by the leadership of the cognitive process, developing the employees' perception of being trusted measurement scale, in order to carry out the employees' perception of being trusted in the empirical research to provide an effective research tool.

\section{THE MOdEL OF THE STRUCTURE OF EMPLOYEES' PERCEPTION OF BEING TRUSTED}

\section{A. Research Methodology}

Qualitative researches have explanatory understanding of existing social phenomena through observation and interviews among other methods, and construct initial theoretical models for deep researches. Grounded theory is a qualitative research method, its analysis method is: conceptualize data through encoding, keep comparing and integrating the conceptual category to depict theoretical framework models. Therefore, this article makes use of interviews to collect first-hand data, and constructs the theoretical model of the inner structure of Chinese employees' perception of being trusted through the grounded theoretical method in qualitative researches.

\section{B. Data Source}

The selection of samples for qualitative researches requires a certain understanding of and contact with the topic of this research on the part of samples. The interviewees hereby should conform to the following requirements: 1 . They come from enterprises; 2 . They have more than half a year's work experience in the same enterprise; 3 . They have a certain level of understanding and expression abilities. 13 interviewees are selected, including 7 employees of state-owned enterprises and 6 employees of private enterprises, differing in terms of their work experience, gender and industry, so the samples are comprehensive. The questions in the interviews this time are 
designed by experts in enterprise management field and qualitative research field, which mainly include "How do you perceive your superiors' trust in you?", "Which specific event or behavior shows superiors' trust in you?" and "Do you feel trusted when superiors have sent signals of trust?", and information is thus obtained. The time of interviewing each interviewee lasts for around 30 minutes, and the interviews are recorded after granting the agreement of the interviewees.

\section{Model Building}

The research makes use of the Chinese version of Nvivo10 software, adopts grounded theory to extract the category of 10 of the 13 interview records, so as to confirm their category, main category and core category, construct theoretical model structure and carry out saturation test on the theory by making use of the remaining three interview records. Extraction of category includes four steps: open coding-axial codingselective coding. (1) Open coding analyzes and reorganizes the content of interviews to extract concepts and categories. For example: the concept of the original words of interviewees "if superiors can leave a large project to you alone, that is a kind of trust in you" is extracted and named as "assigning important tasks". Finally, 32 concepts are extracted, and on this basis, eight categories including authorization, supervision and task assignment, etc. (2) Axial coding weighs the connection and distinctions among eight categories repeatedly by combining the content of interviews, and classifies them according to their attributes, categories with common attributes fall into one main categories. (3) Selective coding chooses core categories, connects them with other categories systematically, and tests their relationship. The relational structure among the main categories in this research is: condition-behavior-result. In other words, the perception of being trust needs certain prerequisites (competence recognition), so that employees will manifest certain behaviors as they are trusted by superiors (set up intimate relationship), and finally lead to the result of being trusted (leadership support). Through generalization, extraction, recombination and integration among other links, the study encodes the core category as "employee perceived trust". The model of the structure of employees' perception of being trusted is shown in Figure 1. The research carries out saturation test on the test by making use of saturation principle to measure the validity of the conceptual models [7]. Through verification, the dimension of structure of the employees' perception of being trusted constructed previously has reached theoretical saturation.

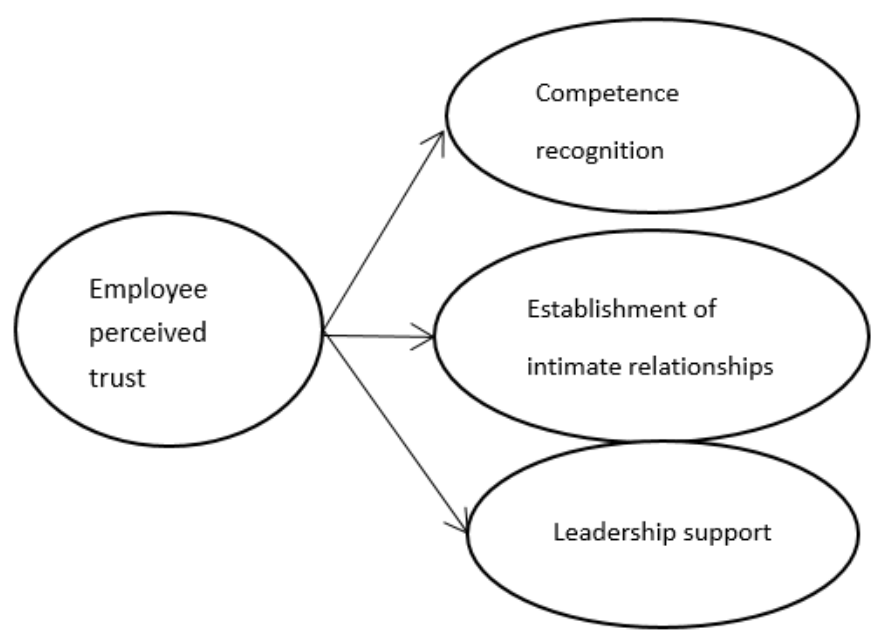

Fig. 1 The model of the structure of employees' perception of being trusted

\section{DEVELOPMENT OF THE SCALE OF THE EMPLOYEES' PERCEPTION OF BEING TRUSTED}

\section{A. The Formation of Official Questionnaires}

\section{1) Formulate the initial scale}

Convert the 32 concepts obtained through qualitative analysis into descriptive behavioral measurement questions, reflecting one specific behavior with each behavior measurement question. Translate foreign scales in a professional way, and repeatedly compare the generalized 32 behavior measurement questions and existing scales, add to or reduce the content, ensure that each question is semantically clear, complete and concise in content, and finally an initial scale of the employees' perception of being trusted including 18 questions is formed.

\section{2) Pre-test and Analysis}

There are two rounds of tests in this research, various organizers and employees are chosen as respondents of both rounds. The questionnaires are handed out through professional online questionnaire platform and on the spot, there are over 90 valid questionnaires. Likert 5-point scoring method is adopted for the questionnaires, the figures of 1 to 5 mean "disagree" to "strongly agree". Exploratory factor analysis is conducted on the data collected with SPASS.20, and the analysis results are verified according to standard indices such as common degree value of various questions being over 0.4 , cross-load value being less than 0.4 , and validity KMO value and reliability coefficient value being over 0.6. Finally, 9 questions are confirmed and official questionnaires are formed for verification.

\section{3) Official Test}

The respondents and way of handing out questionnaires in the official test are the same as those in pre-test. All in all, 250 questionnaires are handed out, and 241 of them are valid, with an effective rate of $96.4 \%$. Divide these questionnaires into two groups for exploratory factor analysis and confirmatory factor analysis. The distribution of the samples of the questionnaires is even and extensive, covering eight industries including finance, trade and service, etc., 


\section{B. Explanatory Factor Analysis of Official Quesionnaires}

Choose 120 questionnaires from the 241 questionnaires randomly in the official test and conduct EFA (Explantory Factory Analysis), through analysis, the KMO value is 0.805 , indicating a high quantitative validity. Three factors are extracted according to the rule that eigenvalue should be larger than 1. Conduct orthogonal rotation for the maximum variance of the component matrix, and the component matrix as shown in Table 1 is obtained.

TABLE I. COMPONENT MATRIX AFTER ROTATION

\begin{tabular}{|c|c|c|c|}
\hline $\begin{array}{l}\text { Item } \\
\text { 1. My superiors allow me to make important decisions } \\
\text { on my own work }\end{array}$ & Factor1 & Factor 2 & $\begin{array}{c}\text { Factor } 3 \\
0.652\end{array}$ \\
\hline $\begin{array}{l}\text { 2. I often have the opportunity to undertake important } \\
\text { work on my own }\end{array}$ & & & 0.795 \\
\hline $\begin{array}{l}\text { 3. My superiors feel assured about the work handed } \\
\text { over to me. }\end{array}$ & & & 0.828 \\
\hline $\begin{array}{l}\text { 4. My superior asks me to accompany him/her to be } \\
\text { present on important occasions }\end{array}$ & & 0.754 & \\
\hline $\begin{array}{l}\text { 5. My superior often discloses to me important } \\
\text { information unknown to others at work }\end{array}$ & & 0.881 & \\
\hline $\begin{array}{l}\text { 6. My superior is willing to discuss with me the } \\
\text { problems or difficulty he/she meets at work }\end{array}$ & & 0.605 & \\
\hline $\begin{array}{l}\text { 7. Even if my thoughts are not comprehensive in } \\
\text { consideration or not mature, my superior will still give } \\
\text { me positive feedback }\end{array}$ & 0.798 & & \\
\hline $\begin{array}{l}\text { 8. My superior gives me truthful suggestions and help } \\
\text { to me at work. }\end{array}$ & 0.867 & & \\
\hline 9. My superior is willing to put himself in my shoe & 0.785 & & \\
\hline Digenvalue (After rotation) & 2.260 & 1.990 & 1.980 \\
\hline Variance interpretation rate $\%$ (after rotation) & $25.107 \%$ & $22.117 \%$ & $22.002 \%$ \\
\hline $\begin{array}{l}\text { Cumulative variance interpretation rate } \% \text { (after } \\
\text { rotation) }\end{array}$ & $25.107 \%$ & $47.224 \%$ & $69.226 \%$ \\
\hline KMO value & 0.805 & & \\
\hline Barlett spherical value & 361.196 & & \\
\hline df & 36 & & \\
\hline$P$ value & 0.000 & & \\
\hline
\end{tabular}

As shown in Table 1, factor 1 reflects leadership's advice and help to employees, so it is named leadership support. Factor 2 reflects the degree of intimacy between leaders and employees, so it is named as establishing intimacy. Factor 3 reflects the size of the employees' autonomy in their work, the importance of their tasks and the degree of confidence of their leaders, therefore it is named as capacity recognition. At this point, the results of exploratory analysis of employees' perceived trust structure are basically the same as those of grounded theory.

\section{Verification Analysis on Official Questionnaires}

The article verifies the structure of the model with the CFA procedures of AMOS21.0. First of all, presume that the employees' perception of being trusted is a first-order threeelement model, and conduct verification analysis on the remaining 121 of the 241 questionnaires. The factor loading of all the observed variables in the three-factor model is over 0.5 , and various fit indices of the first-order mode have met the standards, which means that the three-factor model of the employees' perception of being trusted obtained through exploratory factor analysis is testified. Divide the structure of the employees' perception of being trusted into one dimension, two dimensions and four dimensions and verify the factors (Table 2). Through multi-factor model verification it is concluded that it is most ideal when the employees' perception of being trusted is a three-factor model. Meanwhile, as the estimated covariance of the constructive variables of the three elements has all reached the significant level of 0.001 , the correlation among the three factors in the scale of perception of being trusted is significant, high-order factors can be aggregated, and are thus named as the employees' perception of being trusted.

Since the items involved in each questionnaire are filled in by the same person in the survey, CMV may occur. Therefore, the article adopts Harman's single-factor test method to observe whether there is common method bias [8] in the data. Load the nine questions of the variable on one common factor, and construct single-factor structural function model to test the fitting of the model (See Table 3, the Fitting Index of the Model of First-order CFA of the Employees' Perception of Being Trusted). The fitting of the model is not ideal, which means that there are no severe common method biases in the data of this research. 
TABLE II. The Fitting Index of the Model of First-order CFA of the EMPloyeEs’ Perception of Being TRUSTED

\begin{tabular}{ccccccccccc}
\hline & CMIN & DF & $\begin{array}{c}\text { CMIN } \\
/ \text { DF }\end{array}$ & $\begin{array}{c}\text { RMSE } \\
\text { A }\end{array}$ & IFI & TLI & CFI & PGFI & PNFI & PCFI \\
\hline standard & & & $<3$ & $<0.05$ & $>0.9$ & $>0.9$ & $>0.9$ & $>0.5$ & $>0.5$ & $>0.5$ \\
single-factor model & 91.187 & 27 & 3.377 & 0.141 & 0.842 & 0.785 & 0.839 & 0.503 & 0.592 & 0.629 \\
two-factor model & 50.542 & 26 & 1.944 & 0.089 & 0.940 & 0.915 & 0.938 & 0.521 & 0.638 & 0.678 \\
four-factor model & 22.473 & 24 & 0.936 & 0.000 & 1.004 & 1.006 & 1.000 & 0.513 & 0.632 & 0.667 \\
three-factor model & 33.862 & 21 & 1.612 & 0.071 & 0.969 & 0.945 & 0.968 & 0.440 & 0.538 & 0.564 \\
\hline
\end{tabular}

D. Reliance and Validity Test of the Official Questionnaires

The research adopts Cronbach' Alpha and CR of the potential variables to measure the reliability of the questionnaires, if Cronbach'Alpha is larger than 0.7, the questionnaires have a high reliability; if $\mathrm{CR}$ value is over 0.6 , it means that the internal quality of the model is ideal. In this study, all the dimensions and overall reliability indices of the scale have exceeded general standards, showing the stability and dependability of the questionnaires.

In this research, content validity and structure validity are mainly used for the measurement of the questionnaires. (1) Qualitative analysis is adopted for content validity. Each step in the process of producing these questionnaires is quite scientific and rigorous, and has a high level of content validity. (2) Structural validity verifies the convergent validity and discrimination validity of the questionnaires. The research determines the convergent validity of the questionnaires through AVE value. The results of the test show that, the AVE value of ability recognition, establishing intimacy and leaders' support is respectively $0.557,0.522$ and 0.573 , larger than 0.5 , signifying good convergent validity of the questionnaires. Discrimination validity makes judgment through testing the competitiveness of the models of different factors. From Table 3 it can be known that, compared to single-factor model, twofactor model and four-factor model, three-factor model has the best degree of fitting, showing a relatively good discrimination validity of the questionnaires.

\section{CONCLUSION}

This study found three dimensions of employees' perception of being trusted, including competence recognition, establishment of intimate relationships and leadership support. Competence recognition is mainly embodied in the degree of supervision and assignment of tasks. The establishment of intimate relationships is mainly reflected in the establishment of life and friends, and the support of leadership is mainly reflected in giving help and advice to the staff. The theoretical significance of the development of this scale is that the connotation and function of the dimensions of the scale are more clear, and have typical Chinese cultural characteristics. It provides a research tool for continuing to explore the perception of being trusted of employees in China, and also contributes to the cross culture research of the foreign scholars on the problem of the perception of being trusted.

\section{REFERENCES}

[1] Wang Hongli, Zhang Quanjun. The Cost of Being Trusted: Research on the Relationship among Employees' Perception of Being Trusted by Superiors, Role Load, Work Pressure and Emotional Exhaustion [J]. Management World, 2016 (8):110-125. (In Chinese)

[2] Deng J, Wang K. Feeling Trusted And Loyalty: Modeling SupervisorSubordinate Interaction From A Trustee Perspective [J]. International Employment Relations Review, 2009, 15(1)

[3] Lau D C, Long W L, Wen S S. Examining the effects of feeling trusted by supervisors in the workplace: A self - evaluative perspective [J]. Journal of Organizational Behavior, 2014, 35(1):112-127.

[4] Jon L. Pierce, Donald G. Gardner. Self-Esteem Within the Work and Organizational Context: A Review of the Organization-Based SelfEsteem Literature [J]. Journal of Management, 2004, 30(5):591-622.

[5] Farndale E, Hailey V H, Kelliher C.High Commitment Performance Management: The Roles of Justice and Trust [J]. Personnel Review, 2011, 40(1):5-23.

[6] Wang Hongli, Lv Diwei. Research on the Employees' Perception of Trust and Its Influences with the Perspective of Controlled Motivation Theory [J]. Journal of Management, 2018, 15(3):351-352. (In Chinese)

[7] Pandit N. The creation of theory: A recent application of the grounded theory method [J]. The Qualitative Report, 1996, 2(4):1-20.

[8] Zhou Haolong, Li Rong. Statistical Test and Controlling Method of Common Method Bias [J]. Advances in Psychological Science, 2004, 12(6): 942-950. (In Chinese) 